\title{
Existence and Multiplicity Results of Homoclinic Solutions for the DNLS Equations with Unbounded Potentials
}

\author{
Defang $\mathrm{Ma}^{1,2}$ and Zhan Zhou' ${ }^{1,2}$ \\ ${ }^{1}$ School of Mathematics and Information Science, Guangzhou University, Guangdong, \\ Guangzhou 510006, China \\ ${ }^{2}$ Key Laboratory of Mathematics and Interdisciplinary Sciences of Guangdong Higher Education Institutes, \\ Guangzhou University, Guangdong, Guangzhou 510006, China
}

Correspondence should be addressed to Zhan Zhou, zzhou0321@hotmail.com

Received 25 July 2012; Accepted 9 September 2012

Academic Editor: Wenming Zou

Copyright @ 2012 D. Ma and Z. Zhou. This is an open access article distributed under the Creative Commons Attribution License, which permits unrestricted use, distribution, and reproduction in any medium, provided the original work is properly cited.

A class of difference equations which include discrete nonlinear Schrödinger equations as special cases are considered. New sufficient conditions of the existence and multiplicity results of homoclinic solutions for the difference equations are obtained by making use of the mountain pass theorem and the fountain theorem, respectively. Recent results in the literature are generalized and greatly improved.

\section{Introduction}

Assume that $m$ is a positive integer. Consider the following difference equation in infinite $m$ dimensional lattices,

$$
L u_{n}+v_{n} u_{n}-\omega u_{n}=\sigma f\left(n, u_{n}\right), \quad n \in \mathbb{Z}^{m},
$$

where $\sigma= \pm 1, n=\left(n_{1}, n_{2}, \ldots, n_{m}\right) \in \mathbb{Z}^{m},\left\{u_{n}\right\}$ is a real valued sequence, $\omega \in \mathbb{R}, L$ is a Jacobi operator [1] given by

$$
\begin{aligned}
L u_{n}= & a_{1\left(n_{1}, n_{2}, \ldots, n_{m}\right)} u_{\left(n_{1}+1, n_{2}, \ldots, n_{m}\right)}+a_{1\left(n_{1}-1, n_{2}, \ldots, n_{m}\right)} u_{\left(n_{1}-1, n_{2}, \ldots, n_{m}\right)} \\
& +a_{2\left(n_{1}, n_{2}, \ldots, n_{m}\right)} u_{\left(n_{1}, n_{2}+1, \ldots, n_{m}\right)}+a_{2\left(n_{1}, n_{2}-1, \ldots, n_{m}\right)} u_{\left(n_{1}, n_{2}-1, \ldots, n_{m}\right)}
\end{aligned}
$$




$$
\begin{aligned}
& +\cdots+a_{m\left(n_{1}, n_{2}, \ldots, n_{m}\right)} u_{\left(n_{1}, n_{2}, \ldots, n_{m}+1\right)}+a_{m\left(n_{1}, n_{2}, \ldots, n_{m}-1\right)} u_{\left(n_{1}, n_{2}, \ldots, n_{m}-1\right)} \\
& +b_{\left(n_{1}, n_{2}, \ldots, n_{m}\right)} u_{\left(n_{1}, n_{2}, \ldots, n_{m}\right)}
\end{aligned}
$$

where $\left\{a_{i n}\right\}(i=1,2, \ldots, m)$ and $\left\{b_{n}\right\}$ are real valued and bounded sequences.

We assume that $f(n, 0)=0$ for $n \in \mathbb{Z}^{m}$, then $u_{n}=0$ is a solution of (1.1), which is called the trivial solution. As usual, we say that a solution $u=\left\{u_{n}\right\}$ of (1.1) is homoclinic (to 0$)$ if

$$
\lim _{|n| \rightarrow \infty} u_{n}=0
$$

where $|n|=\left|n_{1}\right|+\left|n_{2}\right|+\cdots+\left|n_{m}\right|$ is the length of multiindex $n$. In addition, if $u_{n} \not \equiv 0$, then $u$ is called a nontrivial homoclinic solution. We are interested in the existence and multiplicity of the nontrivial homoclinic solutions for (1.1). This problem appears when we look for the discrete solitons of the following Discrete Nonlinear Schrödinger (DNLS) equation:

$$
i \dot{\psi}_{n}+\Delta \psi_{n}-v_{n} \psi_{n}+\sigma f\left(n, \psi_{n}\right)=0, \quad n \in \mathbb{Z}^{m},
$$

where

$$
\begin{aligned}
\Delta \psi_{n}= & \psi_{\left(n_{1}+1, n_{2}, \ldots, n_{m}\right)}+\psi_{\left(n_{1}, n_{2}+1, \ldots, n_{m}\right)}+\cdots+\psi_{\left(n_{1}, n_{2}, \ldots, n_{m}+1\right)}-2 m \psi_{\left(n_{1}, n_{2}, \ldots, n_{m}\right)} \\
& +\psi_{\left(n_{1}-1, n_{2}, \ldots, n_{m}\right)}+\psi_{\left(n_{1}, n_{2}-1, \ldots, n_{m}\right)}+\cdots+\psi_{\left(n_{1}, n_{2}, \ldots, n_{m}-1\right)}
\end{aligned}
$$

is the discrete Laplacian in $m$ spatial dimension. Moreover, assume that the nonlinearity $f(n, u)$ is gauge invariant, that is,

$$
f\left(n, e^{i \theta} u\right)=e^{i \theta} f(n, u), \quad \theta \in \mathbb{R}
$$

Since solitons are spatially localized time-periodic solutions and decay to zero at infinity. Thus $\psi_{n}$ has the form

$$
\begin{gathered}
\psi_{n}=u_{n} e^{-i \omega t}, \\
\lim _{|n| \rightarrow \infty} \psi_{n}=0,
\end{gathered}
$$

where $\left\{u_{n}\right\}$ is a real-valued sequence, and $\omega \in \mathbb{R}$ is the temporal frequency. Then (1.4) becomes

$$
-\Delta u_{n}+v_{n} u_{n}-\omega u_{n}=\sigma f\left(n, u_{n}\right), \quad n \in \mathbb{Z}^{m},
$$

and (1.3) holds. Naturally, if we look for solitary solutions of (1.4), we just need to get the homoclinic solutions of (1.8). Obviously, (1.8) is a special case of (1.1) with $a_{i n} \equiv-1(i=$ $1,2, \ldots, m), b_{n} \equiv 2 m$. 
DNLS equation is one of the most important inherently discrete models, which models many phenomena in various areas of applications (see [2-4] and reference therein). For example, in nonlinear optics, DNLS equation appears as a model of infinite wave guide arrays. In the past decade, the existence and properties of mobile discrete solitons/breathers in DNLS equations have been considered in a number of studies [5-9].

When $m=1, v_{n} \equiv 0$, and $\left\{a_{n}\right\},\left\{b_{n}\right\}$, and $f(n, u)$ are $T$-periodic in $n$, the existence of homoclinic solutions for the (1.1) have been studied in $[5,6,10]$ for the case where $f$ is with superlinear nonlinearity (kerr or cubic), in $[9,11-14]$ for the case where $f$ is with saturable nonlinearity, respectively. When $\left\{a_{n}\right\},\left\{b_{n}\right\}$, and $f(n, u)$ are not periodic in $n$, the existence of homoclinic solutions for some special case of (1.1) can be found in $[7,8,15,16]$. Especially, in $[17,18]$, the authors obtained sufficient conditions for the existence of at least a pair of nontrivial homoclinic solutions for the special case of (1.1) when $\left\{v_{n}\right\}$ is unbounded by Nehari manifold method. It is worth pointing out that the so-called global AmbrosettiRabinowitz condition of $f$ plays a crucial role in $[17,18]$. One aim of this paper is to replace the global Ambrosetti-Rabinowitz condition by a general one. The other aim of this paper is to obtain sufficient conditions for the existence of infinitely many nontrivial homoclinic solutions of (1.1). We will see that in Section 2, our results greatly improves those in $[17,18]$. Our proofs of the main results are based on Mountain Pass Lemma and Fountain theorem. Our main ideas come from the papers [19-22].

This paper is organized as follows: in Section 2, we will first define some basic spaces. Then, we give the main results of this paper, and a comparison with the existing results is stated. Third, we establish the variational framework associated with (1.1) and transfer the problem of the existence and multiplicity of solutions in $E$ (defined in Section 2) of (1.1) into that of the existence and multiplicity of critical points of the corresponding functional. We also recall some basic results from critical point theory. Last, in Section 3, we present the proofs of our main results.

\section{Preliminaries and Main Results}

Let

$$
l^{p} \equiv l^{p}\left(\mathbb{Z}^{m}\right)=\left\{u=\left\{u_{n}\right\}_{n \in \mathbb{Z}^{m}}: \forall n \in \mathbb{Z}^{m}, u_{n} \in \mathbb{R},\|u\|_{l^{p}}=\left(\sum_{n \in \mathbb{Z}^{m}}\left|u_{n}\right|^{p}\right)^{1 / p}<\infty\right\}
$$

Then the following embedding between $l^{p}$ spaces holds:

$$
l^{q} \subset l^{p}, \quad\|u\|_{l^{p}} \leq\|u\|_{l^{q}}, \quad 1 \leq q \leq p \leq \infty
$$

Assume the following condition on $\left\{v_{n}\right\}$ holds.

$\left(V_{1}\right)$ the discrete potential $V=\left\{v_{n}\right\}_{n \in \mathbb{Z}^{m}}$ satisfies

$$
\lim _{|n| \rightarrow \infty} v_{n}=\infty
$$


Let

$$
H=L+V
$$

Since the operator $L$ is bounded and self-adjoint in the space $l^{2}\left(\mathbb{Z}^{m}\right)$ with the norm $\|L\|$ (see $[1])$, and by the condition $\left(V_{1}\right)$, we know that the potential $V$ is bounded below, without loss of generality, we suppose $v_{n}>\|L\|$ for all $n \in \mathbb{Z}^{m}$. Then the operator $H$ is an unbounded positive self-adjoint operator in $l^{2}\left(\mathbb{Z}^{m}\right)$.

Define the space

$$
E:=\left\{u \in l^{2}\left(\mathbb{Z}^{m}\right): H^{1 / 2} u \in l^{2}\left(\mathbb{Z}^{m}\right)\right\}
$$

Then $E$ is a Hilbert space equipped with the norm

$$
\|u\|=\left\|H^{1 / 2} u\right\|_{l^{2}\left(Z^{m}\right)}
$$

Since $\left(V_{1}\right)$ holds, we see that the spectrum $\sigma(H)$ is discrete and let $\lambda_{1}$ be the smallest eigenvalue of $H$, that is

$$
\lambda_{1}=\inf \sigma(H)
$$

Now, we present the following basic hypotheses in order to establish the main results in this paper:

$\left(f_{1}\right) f \in C\left(\mathbb{Z}^{m} \times \mathbb{R}, \mathbb{R}\right)$, and there exists $a>0, p \in(2, \infty)$ such that

$$
|f(n, u)| \leq a\left(1+|u|^{p-1}\right), \quad \forall n \in \mathbb{Z}^{m}, u \in \mathbb{R} .
$$

$\left(f_{2}\right) \lim _{|u| \rightarrow 0} f(n, u) / u=0$ uniformly for $n \in \mathbb{Z}^{m}$.

$\left(f_{3}\right) \lim _{|u| \rightarrow \infty} F(n, u) / u^{2}=+\infty$ uniformly for $n \in \mathbb{Z}^{m}$, where $F(n, u)$ is the primitive function of $f(n, u)$, that is,

$$
F(n, u)=\int_{0}^{u} f(n, t) d t
$$

$\left(f_{4}\right) f(n, u) / u$ is increasing in $u>0$ and decreasing in $u<0$, for all $n \in \mathbb{Z}^{m}$.

Under the above hypotheses, our results can be stated as follows.

Theorem 2.1. Assume that conditions $\left(V_{1}\right),\left(f_{1}\right)-\left(f_{4}\right)$ hold. Then, we have the following conclusions.

(1) If $\sigma=-1, \omega \leq \lambda_{1},(1.1)$ has no nontrivial solution in $E$.

(2) If $\sigma=1, \omega<\lambda_{1},(1.1)$ has at least one nontrivial solution $u$ in $E$.

(3) The solutions obtained in case (2) exponentially decay at infinity, that is, there exist two positive constants $C$ and $\alpha$ such that

$$
\left|u_{n}\right| \leq C e^{-\alpha|n|}, \quad n \in \mathbb{Z}^{m} .
$$


Theorem 2.2. Assume $f(n, u)$ is odd in $u$ for each $n \in \mathbb{Z}^{m}$, and that conditions $\left(V_{1}\right),\left(f_{1}\right)-\left(f_{4}\right)$ hold. Then we have the following conclusions.

(1) If $\sigma=-1, \omega \leq \lambda_{1},(1.1)$ has no nontrivial solution in $E$.

(2) If $\sigma=1, \omega<\lambda_{1},(1.1)$ has infinitely many solutions $\left\{u^{(k)}\right\}_{k=1}^{\infty}$ in E satisfying

$$
\frac{1}{2}\left(H u^{(k)}, u^{(k)}\right)-\frac{1}{2} \omega\left(u^{(k)}, u^{(k)}\right)-\sum_{n \in Z^{m}} F\left(n, u_{n}^{(k)}\right) \longrightarrow \infty, \quad \text { as } k \longrightarrow \infty
$$

(3) The solutions obtained in case (2) exponentially decay at infinity, that is, (2.10) holds.

We notice that, in $[17,18]$, the authors consider the following DNLS equation

$$
H u_{n}-\omega u_{n}-\sigma \gamma_{n} f\left(u_{n}\right)=0,
$$

which is a special case of (1.1), where $H=-\Delta+V$. They obtain the following results.

Theorem A. Assume that the DNLS (2.12) satisfies $\left(V_{1}\right)$ and

$\left(A_{1}\right)$ there exist two positive constants $\underline{\gamma}$ and $\bar{\gamma}$, such that for any $n \in \mathbb{Z}^{m}$,

$$
\underline{r} \leq r_{n} \leq \bar{\gamma}
$$

The nonlinearity $f$ is odd and satisfies

$\left(A_{2}\right)$ there are two positive constants $C_{1}, C_{2}$, and $2<p<\infty$ such that

$$
\begin{gathered}
|f(u)| \leq C_{1}\left(1+|u|^{p-1}\right) \\
|f(u)-f(v)| \leq C_{2}\left(1+|u|^{p-2}+|v|^{p-2}\right)|u-v| .
\end{gathered}
$$

$\left(A_{3}\right) \lim _{u \rightarrow 0}(f(u) /|u|)=0$.

$\left(A_{4}\right)$ there is a $2<q<\infty$ such that

$$
0<(q-1) f(u) u \leq f^{\prime}(u) u^{2}, \quad \forall u \neq 0
$$

Then we have the following conclusions.

(1) If $\sigma=-1, \omega \leq \lambda_{1},(2.12)$ has no nontrivial solution.

(2) If $\sigma=1, \omega<\lambda_{1},(2.12)$ has at least a pair of nontrivial solutions $\pm u$ in $l^{2}\left(\mathbb{Z}^{m}\right)$.

(3) The solutions obtained in case (2) exponentially decay at infinity, that is, (2.10) holds.

Remark 2.3. Clearly, (2.12) corresponds (1.1) if we let

$$
L=-\Delta, \quad f(n, u)=\gamma_{n} f(u) .
$$


Equations (2.13) and (2.14) imply $\left(f_{1}\right)$, conditions $\left(A_{1}\right)$ and $\left(A_{3}\right)$ imply $\left(f_{2}\right)$, and conditions $\left(A_{1}\right),\left(A_{4}\right)$ imply $\left(f_{3}\right)$ and $\left(f_{4}\right)$. Equation (2.15) is unnecessary in Theorem 2.2. Thus, our Theorem 2.2 greatly improves Theorem A.

Remark 2.4. In (2.12), we define $f$ by

$$
f(u)= \begin{cases}0, & u=0, \\ \frac{u}{1-\ln |u|}, & 0<|u| \leq 1, \\ u(1+\ln |u|), & |u|>1,\end{cases}
$$

then $f$ does not satisfy $\left(A_{4}\right)$. However, if we let $f(n, u)=\gamma_{n} f(u)$ in (1.1), where $\left\{\gamma_{n}\right\}$ satisfies $\left(A_{1}\right)$, then $f(n, u)$ satisfies all conditions in Theorem 2.2.

Now, we will make some preparations for the proofs of our main results. Since the operator $L$ is bounded in $l^{2}\left(\mathbb{Z}^{m}\right)$, the following two norms are equivalent in the Hilbert space E

$$
\|u\| \sim\left\|V^{1 / 2} u\right\|_{l^{2}\left(\mathbb{Z}^{m}\right)}
$$

The following theorem plays an important role in this paper, which gives a discrete version of compact embedding theorem [16-18].

Lemma 2.5. If $V$ satisfies the condition $\left(V_{1}\right)$, then for any $2 \leq p \leq \infty$, the embedding map from $E$ into $l^{p}\left(\mathbb{Z}^{m}\right)$ is compact, denote the best embedding constant $c_{p}=\max _{\|u\|_{p}=1} 1 /\|u\|$.

Consider the function $J$ defined on $E$ by

$$
J(u)=\frac{1}{2}((H-\omega) u, u)-\sigma \sum_{n \in Z^{m}} F\left(n, u_{n}\right)
$$

Standard arguments show that the functional $J$ is well-defined $C^{1}$ functional on $E$ and (1.1) is easily recognized as the corresponding Euler-Lagrange equation for $J$. Thus, to find nontrivial solutions of (1.1), we need only to look for nonzero critical points of $J$.

For the derivative of $J$ we have the following formula:

$$
\left(J^{\prime}(u), v\right)=((H-\omega) u, v)-\sigma \sum_{n \in Z^{m}} f\left(n, u_{n}\right) v_{n}, \quad \forall v \in E .
$$

Definition 2.6 (see $[22,23]$ ). Let $E$ be a real Banach Space and $J \in C^{1}(E, \mathbb{R})$. For some $c \in \mathbb{R}$, we say $J$ satisfies the so-called $(C)_{c}$ condition if any sequence $\left\{u_{n}\right\} \subset E$ such that $J\left(u_{n}\right) \rightarrow c$ and $\left\|J^{\prime}\left(u_{n}\right)\right\|\left(1+\left\|u_{n}\right\|\right) \rightarrow 0$ as $n \rightarrow \infty$, has a convergent subsequence.

Let $B_{r}$ be the open ball in $H$ with radius $r$ and center 0 , and let $\partial B_{r}$ denote its boundary. In order to obtain the existence of critical points of $J$ on $E$, we cite some basic lemmas from [24], which will be used in the proof of Theorem 2.1. The first is the following Mountain Pass Lemma. 
Lemma 2.7. Let $E$ be a real Banach Space, $J \in C^{1}(E, \mathbb{R})$ satisfies the $(C)_{c}$ condition for any $c>$ $0, J(0)=0$, and

$\left(J_{1}\right)$ There exist $\rho, \sigma>0$ such that $\left.J\right|_{\partial B_{\rho}} \geq \alpha$.

$\left(J_{2}\right)$ There exist $e \in E \backslash B_{\rho}$ such that $J(e) \leq 0$.

Then J has a critical value $c \geq \alpha$.

In order to prove Theorem 2.2, we shall use the following fountain theorem $[23,25,26]$. Let $E$ be a real Banach Space with the norm $\|\cdot\|$ and $E=\overline{\bigoplus_{j \in \mathbb{N}} X_{j}}$ with $\operatorname{dim} X_{j}<\infty$ for any $j \in \mathbb{N}$. Set $Y_{k}=\bigoplus_{j=0}^{k} X_{j}$ and $Z_{k}=\overline{\bigoplus_{j=k}^{\infty} X_{j}}$.

Lemma 2.8. Let $J \in C^{1}(E, \mathbb{R})$ be even. If, for each sufficiently large $k \in \mathbb{N}$, there exists $\rho_{k}>\gamma_{k}>0$ such that

$\left(B_{1}\right) a_{k}:=\max _{u \in Y_{k},\|u\|=\rho_{k}} J(u) \leq 0$.

$\left(B_{2}\right) b_{k}:=\inf _{u \in Z_{k},\|u\|=\gamma_{k}} J(u) \rightarrow \infty, k \rightarrow \infty$.

$\left(B_{3}\right) J$ satisfies the $(C)_{c}$ condition for every $c>0$.

Then $J$ has an unbounded sequence of critical values.

\section{Proofs of Main Results}

Lemma 3.1. Suppose that $\sigma=1, \omega<\lambda_{1},\left(V_{1}\right)$ and $\left(f_{1}\right)-\left(f_{4}\right)$ hold, then we have

(i) there exists $\rho, \alpha>0$ such that $\left.J\right|_{\partial B_{\rho}} \geq \alpha$;

(ii) there exists $e \in E$ such that $J(t e) \rightarrow-\infty$ as $|t| \rightarrow \infty$.

Proof. (i) Let $\epsilon=\left(\lambda_{1}-\omega\right) / 2$. According to $\left(f_{1}\right)$ and $\left(f_{2}\right)$, it is easy to show that, there exists $c_{1}>0$, such that,

$$
|f(n, u)| \leq \epsilon|u|+c_{1}|u|^{p-1}
$$

for all $n \in \mathbb{Z}^{m}$ and $u \in \mathbb{R}$. This, together with the mean value theorem, leads to

$$
|F(n, u)|=|F(n, u)-F(n, 0)|=\left|\int_{0}^{1} f(n, s u) u d s\right| \leq \frac{\epsilon}{2}|u|^{2}+\frac{c_{1}}{p}|u|^{p} .
$$

By (3.2) and the Hölder inquality, it follows that

$$
\begin{aligned}
J(u) & =\frac{1}{2}((H-\omega) u, u)-\sum_{n \in \mathbb{Z}^{m}} F\left(n, u_{n}\right) \\
& \geq \frac{1}{2}\left(\lambda_{1}-\omega\right)\|u\|_{2}^{2}-\left(\frac{\epsilon}{2} \sum_{n \in \mathbb{Z}^{m}}\left|u_{n}\right|^{2}+\frac{c_{1}}{p} \sum_{n \in \mathbb{Z}^{m}}\left|u_{n}\right|^{p}\right) \\
& \geq \frac{1}{4}\left(\lambda_{1}-\omega\right)\|u\|_{2}^{2}-\frac{c_{1}}{p}\|u\|_{2}^{p} .
\end{aligned}
$$


Noting that $p>2$, we obtain the following estimate:

$$
J(u) \geq \frac{1}{8}\left(\lambda_{1}-\omega\right) \rho^{2} \equiv \alpha>0, \quad \forall\|u\|=\rho,
$$

with $\rho=\left[\left(p / 8 c_{1}\right)\left(\lambda_{1}-\omega\right)\right]^{1 /(p-2)}$.

(ii) It follows from $\left(f_{3}\right)$ that for any $M>0$, there exists $\delta=\delta(M)>0$ such that for all $n \in \mathbb{R}^{m},|u| \geq \delta$, we have

$$
F(n, u) \geq M|u|^{2}
$$

Notice that, from $\left(f_{2}\right)$ and $\left(f_{4}\right)$, it is easy to get that

$$
F(n, u)>0, \quad \forall u \neq 0
$$

Let $e \in E$ be the eigenvector of $H$ corresponding to the smallest eigenvalue $\lambda_{1}$, that is to say $\mathrm{He}=\lambda_{1} e$. Then, there exists $N>0$, such that

$$
\sum_{|n| \leq N} e_{n}^{2} \geq \frac{1}{2}\|e\|_{2}^{2}
$$

Let

$$
A^{*}=\left\{n \in \mathbb{Z}^{m}:|n| \leq N, e_{n} \neq 0\right\}
$$

Taking $t$ large enough, such that $\left|t e_{n}\right|>\delta$ for all $n \in A^{*}$, then, in view of (3.5)-(3.7), we have

$$
\begin{aligned}
J(t e) & =\frac{1}{2}\left(\lambda_{1}-\omega\right) t^{2}\|e\|_{2}^{2}-\sum_{n \in \mathbb{Z}^{m}} F\left(n, t e_{n}\right) \\
& \leq \frac{1}{2}\left(\lambda_{1}-\omega\right) t^{2}\|e\|_{2}^{2}-\sum_{n \in A^{*}} F\left(n, t e_{n}\right) \\
& \leq \frac{1}{2}\left(\lambda_{1}-\omega\right) t^{2}\|e\|_{2}^{2}-M t^{2} \sum_{n \in A^{*}} e_{n}^{2} \\
& \leq \frac{1}{2}\left(\lambda_{1}-\omega-M\right) t^{2}\|e\|_{2}^{2} .
\end{aligned}
$$

Taking $M$ sufficiently large, for example, $M \geq 2\left(\lambda_{1}-\omega\right)$, we see that $J(t e) \rightarrow-\infty$ as $|t| \rightarrow \infty$. The proof is completed.

Lemma 3.2. Suppose that $\sigma=1, \omega<\lambda_{1},\left(V_{1}\right),\left(f_{1}\right)-\left(f_{4}\right)$ hold. Then the functional $J$ satisfies the $(C)_{c}$ condition for any $c \in \mathbb{R}$. 
Proof. Let $\left\{u^{(k)}\right\} \subset E$ be a $(C)_{c}$ sequence of $J$, that is,

$$
J\left(u^{(k)}\right) \longrightarrow c, \quad\left\|J^{\prime}\left(u^{(k)}\right)\right\|\left(1+\left\|u^{(k)}\right\|\right) \longrightarrow 0, \quad \text { as } k \longrightarrow \infty .
$$

To prove the functional $J$ satisfies the $(C)_{c}$ condition, first, we prove that $\left\{u^{(k)}\right\}$ is bounded in $E$. In fact, if not, we may assume by contradiction that $\left\|u^{(k)}\right\| \rightarrow \infty$ as $k \rightarrow \infty$. Set $\alpha^{(k)}:=$ $u^{(k)} /\left\|u^{(k)}\right\|$. Up to a sequence, we have

$$
\begin{gathered}
\alpha^{(k)}-\alpha \quad \text { in } E, \\
\alpha^{(k)} \longrightarrow \alpha, \quad \text { in } l^{q}\left(\mathbb{Z}^{m}\right), \quad \text { for } q \geq 2 .
\end{gathered}
$$

Case $1(\alpha \neq 0)$. By $J\left(u^{(k)}\right)=c+o(1)$, where $o(1) \rightarrow 0$ as $k \rightarrow \infty$, we have

$$
c+o(1)=J\left(u^{(k)}\right)=\frac{1}{2}\left\|u^{(k)}\right\|^{2}-\frac{1}{2} \omega\left\|u^{(k)}\right\|_{2}^{2}-\sum_{n \in \mathbb{Z}^{m}} F\left(n, u_{n}^{(k)}\right) .
$$

Noticing that $\|u\|^{2}=(H u, u) \geq \lambda_{1}\|u\|_{2}^{2}$, we divide both sides of (3.13) by $\left\|u^{(k)}\right\|^{2}$ and get

$$
\sum_{n \in \mathbb{Z}^{m}} \frac{F\left(n, u_{n}^{(k)}\right)}{\left\|u^{(k)}\right\|^{2}} \leq\left(\frac{1}{2}+\frac{|\omega|}{2 \lambda_{1}}\right)-\frac{c+o(1)}{\left\|u^{(k)}\right\|^{2}}<+\infty
$$

Let $\Omega=\left\{n \in \mathbb{Z}^{m}: \alpha(n) \neq 0\right\}$, then it follows from (3.12) that

$$
u_{n}^{(k)}=\alpha_{n}^{(k)}\left\|u^{(k)}\right\| \longrightarrow+\infty, \quad \text { as } k \longrightarrow \infty, \text { for } n \in \Omega .
$$

In view of $\left(f_{3}\right)$, we have

$$
\lim _{k \rightarrow \infty} \frac{F\left(n, u_{n}^{(k)}\right)}{\left\|u^{(k)}\right\|^{2}}=\lim _{k \rightarrow \infty} \frac{F\left(n, u_{n}^{(k)}\right)}{\left|u_{n}^{(k)}\right|^{2}}\left|\alpha_{n}^{(k)}\right|^{2} \longrightarrow+\infty, \quad \text { for } n \in \Omega .
$$

Therefore,

$$
\sum_{n \in \mathbb{Z}^{m}} \frac{F\left(n, u_{n}^{(k)}\right)}{\left\|u^{(k)}\right\|^{2}}=\left(\sum_{n \in \Omega}+\sum_{n \notin \Omega}\right) \frac{F\left(n, u_{n}^{(k)}\right)}{\left\|u^{(k)}\right\|^{2}} \geq \sum_{n \in \Omega} \frac{F\left(n, u_{n}^{(k)}\right)}{\left\|u^{(k)}\right\|^{2}} \longrightarrow+\infty .
$$

This contradicts (3.14).

Case $2(\alpha=0)$. We define

$$
J\left(t_{k} u^{(k)}\right):=\max _{t \in[0,1]} J\left(t u^{(k)}\right)
$$


For any $M>4$, let $k$ be large enough such that $\left\|u^{(k)}\right\|>M$ and $\bar{\alpha}^{(k)}:=2 M^{1 / 2} u^{(k)} /\left\|u^{(k)}\right\|=$ $2 M^{1 / 2} \alpha^{(k)}$.

By (3.2), (3.12), and $\alpha=0$, it is clear that

$$
\sum_{n \in \mathbb{Z}^{m}} F\left(n, \bar{\alpha}_{n}^{k}\right) \leq \frac{\epsilon}{2}\|\bar{\alpha}\|_{2}^{2}+\frac{c_{1}}{p}\|\bar{\alpha}\|_{2}^{p} \longrightarrow 0, \quad \text { as } k \longrightarrow \infty
$$

Thus, for $k$ large enough

$$
\begin{aligned}
J\left(t_{k} u^{(k)}\right) & \geq J\left(\bar{\alpha}_{n}^{(k)}\right) \\
& =\frac{1}{2}\left\|\bar{\alpha}^{(k)}\right\|^{2}-\frac{1}{2} \omega\left\|\bar{\alpha}^{(k)}\right\|_{2}^{2}-\sum_{n \in \mathbb{Z}^{m}} F\left(n, \bar{\alpha}_{n}^{(k)}\right) \\
& \geq \frac{1}{2}\left\|\bar{\alpha}^{(k)}\right\|^{2}-\frac{1}{2} \omega_{0}\left\|\bar{\alpha}^{(k)}\right\|_{2}^{2}-\sum_{n \in \mathbb{Z}^{m}} F\left(n, \bar{\alpha}_{n}^{(k)}\right) \\
& \geq \frac{1}{2}\left(1-\frac{\omega_{0}}{\lambda_{1}}\right)\left\|\bar{\alpha}^{(k)}\right\|^{2}-\sum_{n \in \mathbb{Z}^{m}} F\left(n, \bar{\alpha}_{n}^{(k)}\right) \\
& \geq\left(1-\frac{\omega_{0}}{\lambda_{1}}\right) M,
\end{aligned}
$$

where $\omega_{0}=\max \{\omega, 0\}<\lambda_{1}$.

This implies that $\lim _{k \rightarrow \infty} J\left(t_{k} u^{(k)}\right)=\infty$. Since $J(0)=0$ and $J\left(u^{(k)}\right) \rightarrow c$ as $k \rightarrow \infty$, $J\left(t u^{(k)}\right)$ attains its maximum at $t_{k} \in(0,1)$ for large $k$. Thus, $\left(J^{\prime}\left(t_{k} u^{(k)}\right), u^{(k)}\right)=0$.

On the other hand, from $\left(f_{4}\right)$, we have that

$$
G(n, s) \leq G(n, t), \quad \forall 0 \leq s<t \quad \text { or } \quad t<s \leq 0, n \in \mathbb{Z}^{m}
$$

where $G(n, t)=f(n, t) t / 2-F(n, t)$. In fact, for $0<s<t$ or $t<s<0$, we have

$$
\begin{aligned}
G(n, t)-G(n, s) & =\frac{t f(n, t)}{2}-\frac{s f(n, s)}{2}-\int_{s}^{t} f(n, \tau) d \tau \\
& \geq \frac{t f(n, t)}{2}-\frac{s f(n, s)}{2}-\frac{f(n, t)}{t} \int_{s}^{t} \tau d \tau \\
& \geq \frac{s^{2}}{2}\left(\frac{f(n, t)}{t}-\frac{f(n, s)}{s}\right)>0 .
\end{aligned}
$$

If $s=0,(3.21)$ is obvious. 
By (3.10) and (3.21), we have

$$
\begin{aligned}
J\left(t_{k} u^{(k)}\right)-\frac{1}{2}\left(J^{\prime}\left(t_{k} u^{(k)}\right), t_{k} u^{(k)}\right) & =\sum_{n \in \mathbb{Z}^{m}}\left(\frac{1}{2} f\left(n, t_{k} u_{n}^{(k)}\right) t_{k} u_{n}^{(k)}-F\left(n, t_{k} u_{n}^{(k)}\right)\right) \\
& \leq \sum_{n \in \mathbb{Z}^{m}}\left(\frac{1}{2} f\left(n, u_{n}^{(k)}\right) u_{n}^{(k)}-F\left(n, u_{n}^{(k)}\right)\right) \\
& =J\left(u^{(k)}\right)-\frac{1}{2}\left(J^{\prime}\left(u^{(k)}\right), u^{(k)}\right) \longrightarrow c, \quad \text { as } k \longrightarrow \infty
\end{aligned}
$$

But (3.20) implies that

$$
J\left(t_{k} u^{(k)}\right)-\frac{1}{2}\left(J^{\prime}\left(t_{k} u^{(k)}\right), t_{k} u^{(k)}\right)=J\left(t_{k} u^{(k)}\right) \longrightarrow \infty
$$

Thus, we get a contradiction. Combining the above arguments, we know that $\left\{u^{(k)}\right\}$ is bounded in $E$.

Second, we show that there is a convergent subsequence of $\left\{u^{(k)}\right\}$. Actually, there exists a subsequence, still denoted by the same notation, such that $u^{(k)}$ weakly converges to some $u \in E$. Applying Lemma 2.5, we see that that, for any $2 \leq q \leq \infty$,

$$
u^{(k)} \longrightarrow u, \quad \text { in } l^{q}\left(\mathbb{Z}^{m}\right)
$$

By a straightforward calculation, we have

$$
\begin{aligned}
\| u^{(k)} & -u\left\|^{2}-\omega\right\| u^{(k)}-u \|_{2}^{2} \\
& =\left(J^{\prime}\left(u^{(k)}\right)-J^{\prime}(u),\left(u^{(k)}-u\right)\right)+\sum_{n \in \mathbb{Z}^{m}}\left(f\left(n, u_{n}^{(k)}\right)-f\left(n, u_{n}\right)\right)\left(u_{n}^{(k)}-u_{n}\right) .
\end{aligned}
$$

Due to the weak convergence, it is clear that the first term $\left(J^{\prime}\left(u^{(k)}\right)-J^{\prime}(u),\left(u^{(k)}-u\right)\right) \rightarrow$ 0 as $k \rightarrow \infty$. It remains to show the second term in the right hand of equality (3.26) also tends to zero as $k \rightarrow \infty$.

Indeed, according to (2.2) and Hölder inequality, we have

$$
\begin{aligned}
& \sum_{n \in \mathbb{Z}^{m}}\left(f\left(n, u_{n}^{(k)}\right)-f\left(n, u_{n}\right)\right)\left(u_{n}^{(k)}-u_{n}\right) \\
& \quad \leq \sum_{n \in \mathbb{Z}^{m}}\left[\epsilon\left(\left|u_{n}^{(k)}\right|+\left|u_{n}\right|\right)+c_{1}\left(\left|u_{n}^{(k)}\right|^{p-1}+\left|u_{n}\right|^{p-1}\right)\right]\left(u_{n}^{(k)}-u_{n}\right) \\
& \quad \leq \epsilon\left(\left\|u^{(k)}\right\|_{2}+\|u\|_{2}\right)\left\|u^{(k)}-u\right\|_{2}+c_{1}\left(\left\|u^{(k)}\right\|_{p}^{p-1}+\|u\|_{p}^{p-1}\right)\left\|u^{(k)}-u\right\|_{p} .
\end{aligned}
$$


Therefore, combining (3.25) and the boundedness of $\left\{u^{(k)}\right\}$, the above inequality implies

$$
\sum_{n \in \mathbb{Z}^{m}}\left(f\left(n, u_{n}^{(k)}\right)-f\left(n, u_{n}\right)\right)\left(u_{n}^{(k)}-u_{n}\right) \longrightarrow 0, \quad \text { as } k \longrightarrow \infty \text {. }
$$

So, from (3.26) we can conclude that $\left\{u^{(k)}\right\} \rightarrow u$ in $E$, and this means $J$ satisfies $(C)_{c}$ condition. The proof is completed.

Proof of Theorem 2.1. (1) By way of contradiction, assume that (1.1) has a nontrivial solution $u \in E$. Then $u$ is a nonzero critical point of $J$ in $E$. Thus, $J^{\prime}(u)=0$. But

$$
\left(J^{\prime}(u), u\right)=((H-\omega) u, u)-\sigma \sum_{n \in \mathbb{Z}^{m}} f\left(n, u_{n}\right) u_{n} \geq \sum_{n \in \mathbb{Z}^{m}} f\left(n, u_{n}\right) u_{n}>0 .
$$

This is a contradiction, so the conclusion holds.

(2) By Lemma 3.1, the functional $J$ satisfies $\left(J_{1}\right)$ and $\left(J_{2}\right)$ of the mountain pass theorem. Lemma 3.2 implies that $J$ satisfies $(C)_{c}$ condition for any $c \in \mathbb{R}$. It follows from Lemma 2.7 that $J$ has a critical value $c \geq \alpha>0$. Hence, (1.1) has at least one nontrivial solution $u \in E$.

(3) Assume $w$ is a nontrivial solution obtained in (2). Similar to [17], let $\Theta=\left\{\theta_{n} \equiv\right.$ $\left.-\sigma f\left(n, w_{n}\right) / w_{n}\right\}$ and the operator $G$ defined by $(G u)_{n}=(H u)_{n}+\theta_{n} u_{n}$ for $u \in E$, then (1.1) is equivalent to

$$
G u=\omega u .
$$

Since $w \in E$, we have $\lim _{|n| \rightarrow \infty} \theta_{n}=0$. The multiplication operator $\Theta$ is compact in $E \subset l^{2}\left(\mathbb{Z}^{m}\right)$, and this implies that

$$
\sigma_{\mathrm{ess}}(G)=\sigma_{\mathrm{ess}}(H)=\phi
$$

Equation (2.10) follows from the standard theorem on exponential decay for the eigenfunction of Jacobi operator (see [1] for details).

This completes the proof of Theorem 2.1.

Assume that $i \in \mathbb{Z}^{m}$. Define $e^{(i)}=\left\{e_{n}^{(i)}\right\}$ by

$$
e_{n}^{(i)}= \begin{cases}1, & n=i \\ 0, & n \neq i\end{cases}
$$

Let $X_{j}=\operatorname{span}\left\{e^{(n)}:|n|=j\right\}$ for $j \in \mathbb{N}=\{0,1,2, \ldots\}, Y_{k}=\bigoplus_{j=0}^{k} X_{j}$, and $Z_{k}=\overline{\bigoplus_{j=k}^{\infty} X_{j}}$, then we have

Lemma 3.3. Suppose that $\sigma=1, \omega<\lambda_{1},\left(V_{1}\right),\left(f_{1}\right)-\left(f_{3}\right)$ hold. Then there exists $\rho_{k}>\gamma_{k}>0$ such that

(i) $b_{k}:=\inf _{u \in Z_{k},\|u\|=\gamma_{k}} J(u) \rightarrow \infty$, as $k \rightarrow \infty$,

(ii) $a_{k}:=\max _{u \in Y_{k},\|u\|=\rho_{k}} J(u) \leq 0$. 
Proof. (i) It follows from (3.2) that, for any $u \in Z_{k}$

$$
\begin{aligned}
J(u) & =\frac{1}{2}(H u, u)-\frac{1}{2} \omega(u, u)-\sum_{n \in \mathbb{Z}^{m}} F\left(n, u_{n}\right) \\
& \geq \frac{1}{2}\|u\|^{2}-\frac{1}{2}(\omega+\epsilon)\|u\|_{2}^{2}-\frac{c_{1}}{p}\|u\|_{p}^{p} \\
& \geq \frac{1}{2}\left[1-(|\omega|+\epsilon) \beta_{k}^{2}\right]\|u\|^{2}-\frac{c_{1}}{p} \beta_{k}^{p}\|u\|^{p},
\end{aligned}
$$

where $\beta_{k}=\sup _{u \in Z_{k},\|u\|=1}\|u\|_{2}$. Since for $u \in Z_{k}$,

$$
\|u\|^{2}=(H u, u)=(L u, u)+(V u, u) \geq-\|L\|\|u\|_{2}^{2}+\sum_{|n| \geq k} v_{n} u_{n}^{2} \geq \inf _{|n| \geq k}\left(v_{n}-\|L\|\right)\|u\|_{2}^{2}
$$

we see that $\beta_{k} \rightarrow 0$ as $k \rightarrow \infty$. Thus,

$$
\begin{aligned}
b_{k} & :=\inf _{u \in Z_{k},\|u\|=\gamma_{k}} J(u) \\
& \geq \inf _{u \in Z_{k},\|u\|=\gamma_{k}} \frac{1}{2}\left[1-(|\omega|+\epsilon) \beta_{k}^{2}\right]\|u\|^{2}-\frac{c_{1}}{p} \beta_{k}^{p}\|u\|^{p} \\
& =\frac{1}{4}\left[1-(|\omega|+\epsilon) \beta_{k}^{2}\right] \gamma_{k}^{2},
\end{aligned}
$$

where $\gamma_{k}=\left[p\left(1-|\omega| \beta_{k}^{2}-\epsilon \beta_{k}^{2}\right) / 4 c_{1} \beta_{k}^{p}\right]^{1 /(p-2)}$. Notice that $p>2$ and $\beta_{k} \rightarrow 0$ as $k \rightarrow \infty$, so we have $b_{k} \rightarrow \infty$, as $k \rightarrow \infty$.

(ii) For any $k \in \mathbb{N}$, let the dimension of $Y_{k}$ be $\chi_{k}$ and $v_{k}^{*}=\max _{|j| \leq k}\left\{v_{j}\right\}$. Assume that $M=\|L\|+v_{k}^{*}-\omega$, then from (3.5), there exists a $\delta_{k}>0$ such that $F(n, u) \geq M|u|^{2}$ for $|u| \geq \delta_{k}$. Thus, for $u \in Y_{k}$,

$$
\begin{aligned}
J(u) & =\frac{1}{2}((H-\omega) u, u)-\sum_{|n| \leq k} F\left(n, u_{n}\right) \\
& =\frac{1}{2}((L+V-\omega) u, u)-\sum_{\left|u_{n}\right| \leq \delta_{k}} F\left(n, u_{n}\right)-\sum_{\left|u_{n}\right|>\delta_{k}} F\left(n, u_{n}\right) \\
& \leq \frac{1}{2}\left(\|L\|+v_{k}^{*}-\omega\right)\|u\|_{2}^{2}-M \sum_{\left|u_{n}\right|>\delta_{k}} u_{n}^{2} \\
& \leq \frac{1}{2}\left(\|L\|+v_{k}^{*}-\omega\right)\|u\|_{2}^{2}-M \sum_{|n| \leq k} u_{n}^{2}+M \sum_{\left|u_{n}\right| \leq \delta_{k}} u_{n}^{2} \\
& \leq \frac{1}{2}\left(\|L\|+v_{k}^{*}-\omega-2 M\right)\|u\|_{2}^{2}+M \delta_{k}^{2} X_{k} \\
& =-\frac{1}{2}\left(\|L\|+v_{k}^{*}-\omega\right)\|u\|_{2}^{2}+\left(\|L\|+v_{k}^{*}-\omega\right) \delta_{k}^{2} X_{k} .
\end{aligned}
$$


Taking $\rho_{k}$ sufficiently large, we have,

$$
a_{k}:=\max _{u \in Y_{k},\|u\|=\rho_{k}} J(u) \leq 0
$$

The proof is completed.

Proof of Theorem 2.2. The proofs for (1) and (3) are similar to that of (1) and (3) in Theorem 2.1, and we omit them. Now we give the proof of (2). By Lemma 3.3, the functional $J$ satisfies $\left(B_{1}\right)$ and $\left(B_{2}\right)$ of Lemma 2.8. Lemma 3.2 implies that $J$ satisfies $(C)_{c}$ condition for any $c \in \mathbb{R}$. $f$ is odd implies that $J(u)$ is even. It follows from Lemma 2.8 that $J$ has a sequence of critical points $\left\{u^{(k)}\right\} \subset E$, such that $J\left(u^{(k)}\right) \rightarrow \infty$. Hence, (1.1) has infinitely many high-energy solutions in $l^{2}\left(\mathbb{Z}^{m}\right)$. This completes the proof.

\section{Acknowledgments}

The authors are grateful to the anonymous referee for his/her valuable suggestions. This work is supported by the National Natural Science Foundation of China (no. 11171078) and the Specialized Fund for the Doctoral Program of Higher Education of China (no. 20114410110002).

\section{References}

[1] G. Teschl, Jacobi Operators and Completely Integrable Nonlinear Lattices, vol. 72 of Mathematical Surveys and Monographs, American Mathematical Society, Providence, RI, USA, 2000.

[2] S. Flach and A. Gorbach, "Discrete breakers-Advances in theory and applications," Physics Reports, vol. 467, pp. 1-116, 2008.

[3] S. Flach and C. R. Willis, “Discrete breathers," Physics Reports, vol. 295, no. 5, pp. 181-264, 1998.

[4] D. Hennig and G. P. Tsironis, "Wave transmission in nonlinear lattices," Physics Reports, vol. 307, no. 5-6, pp. 333-432, 1999.

[5] G. Chen and S. Ma, "Discrete nonlinear Schrödinger equations with superlinear nonlinearities," Applied Mathematics and Computation, vol. 218, no. 9, pp. 5496-5507, 2012.

[6] A. Pankov, "Gap solitons in periodic discrete nonlinear Schrödinger equations," Nonlinearity, vol. 19, no. 1, pp. 27-40, 2006.

[7] A. Pankov, "Standing waves for discrete non-linear Schrödinger equations: sign-changing nonlinearities," Applicable Analysis. In press.

[8] G. Zhang and A. Pankov, "Standing wave solutions of the discrete non-linear Schrödinger equations with unbounded potentials-II," Applicable Analysis, vol. 89, no. 9, pp. 1541-1557, 2010.

[9] Z. Zhou, J. Yu, and Y. Chen, "Homoclinic solutions in periodic difference equations with saturable nonlinearity," Science China Mathematics, vol. 54, no. 1, pp. 83-93, 2011.

[10] A. Pankov, "Gap solitons in periodic discrete nonlinear Schrödinger equations-II. A generalized Nehari manifold approach," Discrete and Continuous Dynamical Systems. Series A, vol. 19, no. 2, pp. 419-430, 2007.

[11] A. Pankov, "Gap solitons in periodic discrete nonlinear Schrödinger equations with saturable nonlinearities," Journal of Mathematical Analysis and Applications, vol. 371, no. 1, pp. 254-265, 2010.

[12] A. Pankov and V. Rothos, "Periodic and decaying solutions in discrete nonlinear Schrödinger with saturable nonlinearity," Proceedings of The Royal Society of London. Series A, vol. 464, no. 2100, pp. 32193236, 2008.

[13] Z. Zhou and J. Yu, "On the existence of homoclinic solutions of a class of discrete nonlinear periodic systems," Journal of Differential Equations, vol. 249, no. 5, pp. 1199-1212, 2010.

[14] Z. Zhou, J. Yu, and Y. Chen, "On the existence of gap solitons in a periodic discrete nonlinear Schrödinger equation with saturable nonlinearity," Nonlinearity, vol. 23, no. 7, pp. 1727-1740, 2010. 
[15] A. Pankov and G. Zhang, "Standing wave solutions for discrete nonlinear Schrödinger equations with unbounded potentials and saturable nonlinearity," Journal of Mathematical Sciences, vol. 177, no. 1, pp. 71-82, 2011.

[16] G. Zhang and A. Pankov, "Standing waves of the discrete nonlinear Schrödinger equations with growing potentials," Communications in Mathematical Analysis, vol. 5, no. 2, pp. 38-49, 2008.

[17] G. Zhang, "Breather solutions of the discrete nonlinear Schrödinger equations with unbounded potentials," Journal of Mathematical Physics, vol. 50, no. 1, pp. 1-12, 2009.

[18] G. Zhang and F. Liu, "Existence of breather solutions of the DNLS equations with unbounded potentials," Nonlinear Analysis. Theory, Methods E Applications, vol. 71, no. 12, pp. e786-e792, 2009.

[19] Y. Ding and C. Lee, "Multiple solutions of Schrödinger equations with indefinite linear part and super or asymptotically linear terms," Journal of Differential Equations, vol. 222, no. 1, pp. 137-163, 2006.

[20] Q. Li, H. Su, and Z. Wei, "Existence of infinitely many large solutions for the nonlinear SchrödingerMaxwell equations," Nonlinear Analysis. Theory, Methods \& Applications, vol. 72, no. 11, pp. 4264-4270, 2010.

[21] D. S. Moschetto, "Existence and multiplicity results for a nonlinear stationary Schrödinger equation," Annales Polonici Mathematici, vol. 99, no. 1, pp. 39-43, 2010.

[22] M.-H. Yang and Z.-Q. Han, “Existence and multiplicity results for the nonlinear Schrödinger-Poisson systems," Nonlinear Analysis. Real World Applications, vol. 13, no. 3, pp. 1093-1101, 2012.

[23] M. Willem, Minimax Theorems, Birkhäuser, Boston, Mass, USA, 1996.

[24] P. Rabinowitz, "Minimax methods in critical point theory with applications to differential equations," vol. 65 of CBMS Regional Conference Series in Mathematics, American Mathematical Society, Providence, RI, USA, 1986.

[25] T. Bartsch, "Infinitely many solutions of a symmetric Dirichlet problem," Nonlinear Analysis. Theory, Methods \& Applications, vol. 20, no. 10, pp. 1205-1216, 1993.

[26] W. Zou, "Variant fountain theorems and their applications," Manuscripta Mathematica, vol. 104, no. 3, pp. 343-358, 2001. 


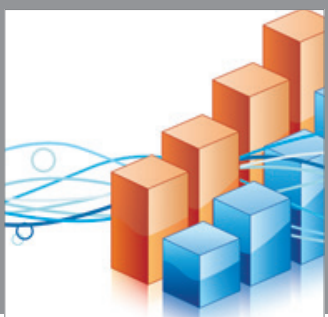

Advances in

Operations Research

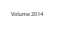

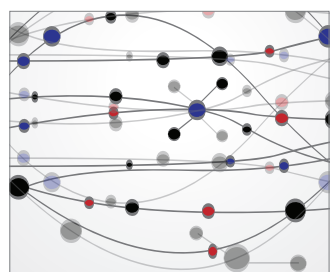

\section{The Scientific} World Journal
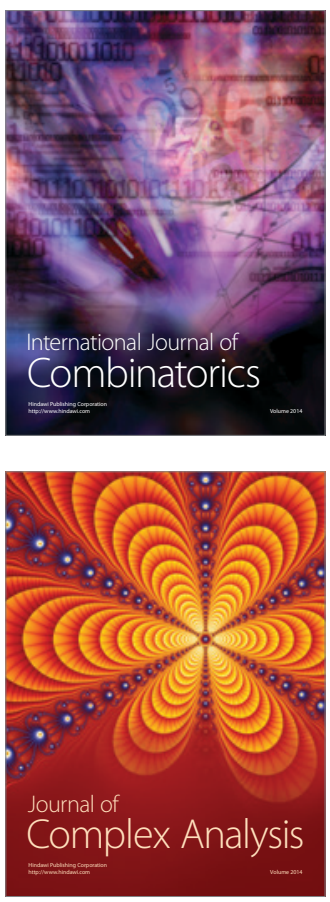

International Journal of

Mathematics and

Mathematical

Sciences
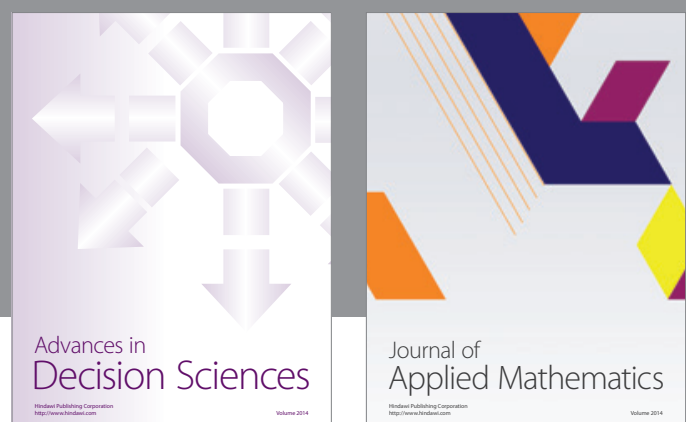

Journal of

Applied Mathematics
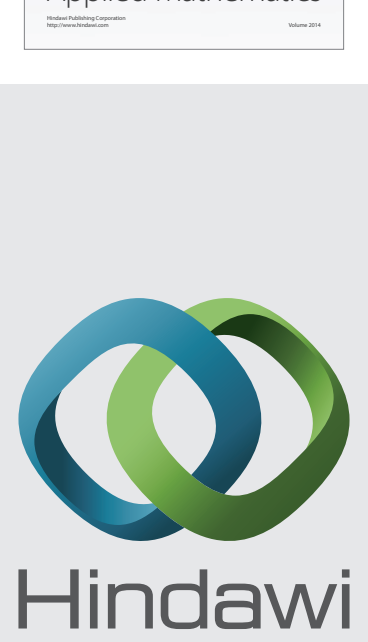

Submit your manuscripts at http://www.hindawi.com
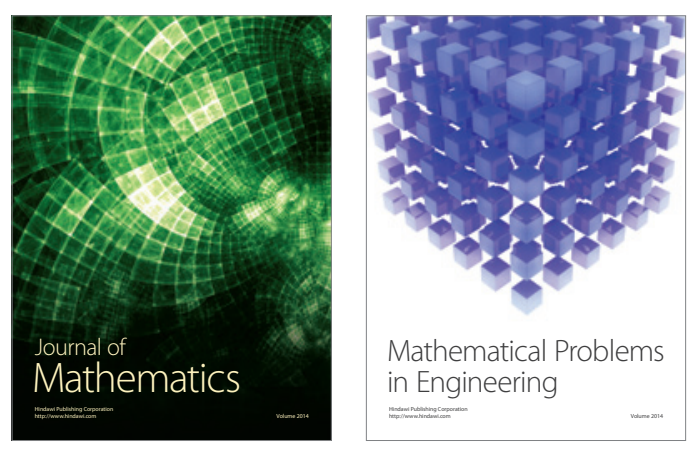

Mathematical Problems in Engineering
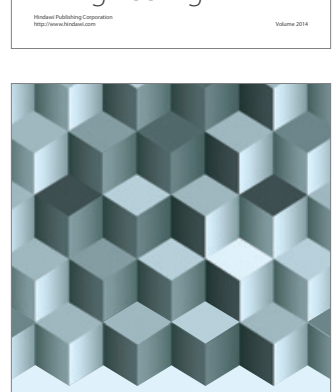

Journal of

Function Spaces
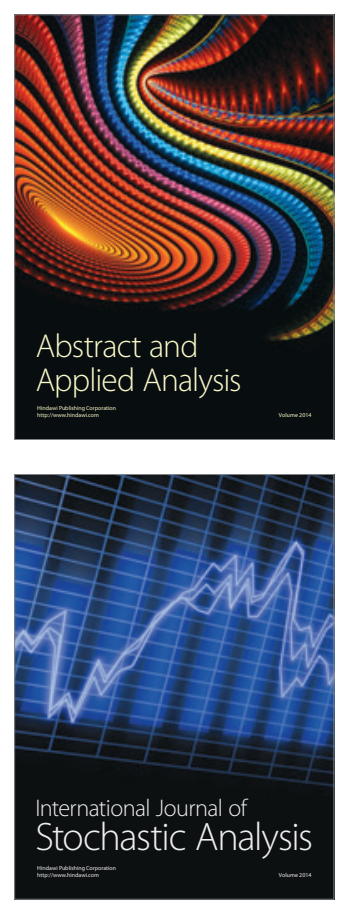

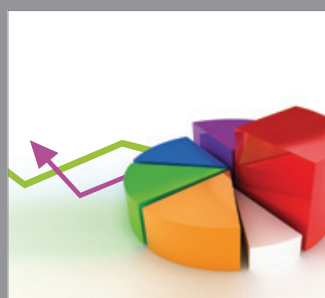

ournal of

Probability and Statistics

Promensencen
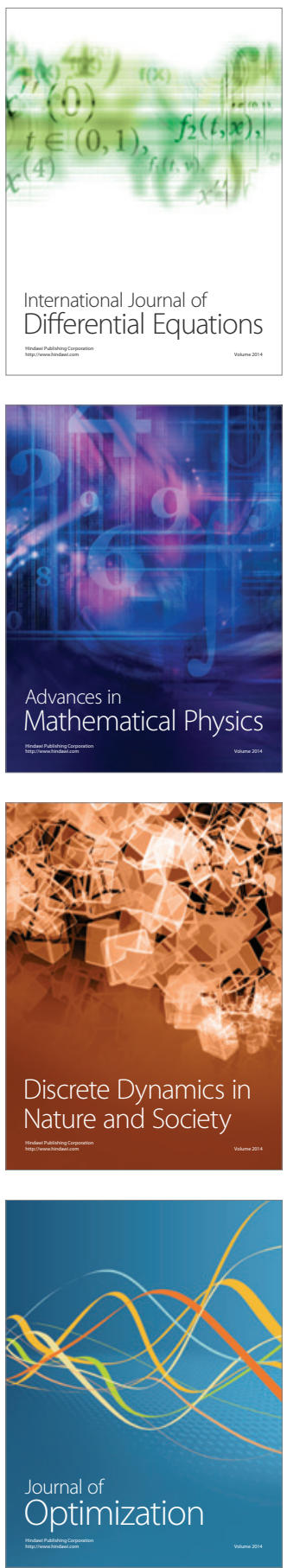